\section{Split Space-Marching Finite-Volume Method for Chemically Reacting Supersonic Flow}

\author{
Arthur W. Rizzi* and Harry E. Bailey $\dagger$ \\ NASA Ames Research Center, Moffett Field, Calif.
}
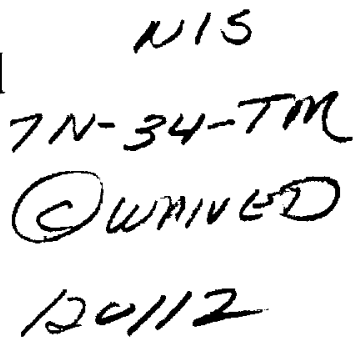

\begin{abstract}
A space-marching finite-volume method employing a nonorthogonal coordinate system and using a split differencing scheme for calculating steady supersonic flow over aerodynamic shapes is presented. It is a secondorder-accurate mixed explicit-implicit procedure that solves the inviscid adiabatic and nondiffusive equations for chemically reacting flow in inlegral conservation-law form. The relationship between the finite-volume and differential forms of the equations is examined and the relative merits of each discussed. The method admits initial Cauchy data situated on any arbitrary surface and integrates them forward along a general curvilinear coordinate, distorting and deforming the surface as it advances. The chemical kinetics term is split from the convective terms which are themselves dimensionally split, thereby freeing the fluid operators from the restricted step size imposed by the chemical reactions and increasing the computational efficiency. The accuracy of this splitting technique is analyzed, a sufficient stability criterion is established, a representative flow computation is discussed, and some comparisons are made with another method.
\end{abstract}

\section{Introduction}

$\mathbf{C}$ ONTINUING interest in and the usefulness of more sophisticated re-entry vehicles than those currently developed has culminated in the decision to design and construct a reusable space shuttle orbiter. This decision has rekindled the need for computing flows with finite-rate chemical reactions. The calculation of such flows is extremely useful to the vehicle designer as a source of information relative to the prediction of heat-transfer rates, boundary-layer effects, and aerodynamic loads acting on the aircraft. At moderate supersonic velocities and lower altitudes the airflow remains in chemical equilibrium, and design information can be obtained from the flow calculations as well as wind-tunnel testing of appropriately scaled models. At higher velocities and altitudes, however, significant nonequilibrium effects are expected to persist over a large area of the vehicle; in addition to affecting the surface heating rate, the dissociating flow produces atomic oxygen which might enhance the oxidation and erosion process at the surface. Complex chemically reacting flow phenomena cannot be scaled, and since current test facilities are incapable of carrying out full-scale experiments in this regime the designer can only turn to the computer solution for a realistic description of the flowfield at actual flight conditions.

For some time numerous procedures ${ }^{1-4}$ for the calculation of one- and two-dimensional inviscid nonequilibrium flow have been known. Our discussion, however, is restricted to those techniques that take advantage of the hyperbolic character of the steady governing equations to integrate initial Cauchy data in a coordinate direction along which the local velocity component is everywhere supersonic. The method of characteristics has been applied recently to reacting flow in three-dimensional nozzles ${ }^{5}$ as well as around general-shaped bodies $^{6}$; more recently a method for calculating reacting flow around an arbitrary body using a finite-difference procedure was introduced by Davy and Reinhardt. ${ }^{7}$

Common to all these existing techniques is the requirement that the initial data lie on a surface symmetrically oriented with respect to the marching coordinate, and the method of

Presented at the AIAA 2nd Computational Fluid Dynamics Conference, Hartford, Conn., June 18-19, 1975; submitted June 20, 1975; revision received November 5,1975 . The authors express their appreciation to Robert MacCormack of Ames Research Center for his helpful suggestions and affable assistance throughout the course of this work.

Index categories: Supersonic and Hypersonic Flow; Reactive Flows.

${ }^{*}$ Research Scientist. Member AIAA.

$\nmid$ †esearch Scientist.
Davy and Reinhardt even further stipulates that this initial surface be a plane normal to the body axis and that it advance downstream and undistorted. This condition simplifies the coordinate geometry and difference equations but severely restricts the choice of acceptable initial data and ignores the orientation of their cones of influence. For flow of a perfect gas Rizzi, et al. ${ }^{8}$ have devised a more general procedure that admits initial conditions situated on any arbitrary surface and integrates them forward along a general curvilinear coordinate that nearly conforms to the local streamline, rotating and distorting the initial data surface as it advances. Adopting that generalized marching method, this paper presents a method for computing the steady flow of an air mixture of dissociating and chemically nonequilibrated gases past a three-dimensional body. The theoretical description of the flow is idealized to the extent that diffusion, heat conduction, and viscous effects are considered negligible.

In addition to increasing the number of equations needed to describe it, the chemical phenomena further complicate the numerical solution by introducing a second intrinsic rate process requiring a step size that can be quite different from that for convection alone. This paper proposes an efficient way of handling these two inherently different rates by developing finite-difference operators that split one spatial dimension from another as well as from the chemical kinetics term and that solve the governing equations to second-order accuracy. The convective operators use an explicit scheme, whereas the chemistry operator is implicit. An analysis of the accuracy of these split operators is carried out, and the computational efficiency gained by them is discussed.

The initial data surface advancing downstream determines the mesh network for the next step, which is aligned with both the bow shock wave and the body surface. This alignment simplifies imposition of the boundary condition at both the shock and body, and ensures consistency with the interior flow at these points.

\section{Continuum Model}

The description of high-temperature airflow past a vehicle involves the calculation not only of the bulk-flow properties of every fluid element, but also the chemical phenomena that the individual gas species comprising the mixture are subjected to within the element. The chemical kinetics of air in the temperature range considered here, mainly entails five species $-\mathrm{O}_{2}, \mathrm{~N}_{2}, \mathrm{~N}, \mathrm{O}$, and $\mathrm{NO}$-entering in the three dissociation reactions and the two bimolecular exchange reactions written in Table 1 . In these reactions $M$ is the third-body collision partner that can be any of the five species in the mix- 
Table 1 Chemical reactions

\begin{tabular}{ll}
\hline \hline$\ell$ & Chemical equation \\
\hline 1 & $\mathrm{O}_{2}+\mathrm{M}=2 \mathrm{O}+\mathrm{M}$ \\
2 & $\mathrm{~N}+\mathrm{M}=2 \mathrm{~N}+\mathrm{M}$ \\
3 & $\mathrm{NO}+\mathrm{M}=\mathrm{N}+\mathrm{O}+\mathrm{M}$ \\
4 & $\mathrm{O}_{2}+\mathrm{N}=\mathrm{NO}+\mathrm{O}$ \\
5 & $\mathrm{~N}_{2}+\mathrm{O}=\mathrm{NO}+\mathrm{N}$ \\
\hline
\end{tabular}

ture. The equations of motion for reacting flow must effectively combine the microscopic formulation of chemical kinetics with the macroscopic equations of gas dynamics governing the bulk-flow properties. The appropriate description for inviscid and nondiffusive flow of a mixture of reacting gases in chemical nonequilibrium is the system of continuum equations that incorporate these two diverse aspects into an overall macroscopic formulation. They are presented here without further discussion. Readers interested in their development and justification are referred to the textbook by Vincenti and Krueger. ${ }^{2}$

The continuity equation for the mixture of density $\rho$ is

$$
\operatorname{div} \rho v=0
$$

and the momentum equation takes the form

$$
\operatorname{div}(\rho v v+p \bar{I})=0
$$

where $p$ signifies the macroscopic pressure, $v$ the bulk velocity, and $\bar{I}$ is the identity tensor. The energy equation with enthalpy $h$ may be written

$$
\operatorname{div}\left[\rho v\left(h+1 / 2 v^{2}\right)\right]=0
$$

and can be integrated at once to obtain $h+1 / 2 v^{2}=$ constant along a streamline. For flow with no spatial variation in the freestream, the only case treated here, the constant is the same for all streamlines and this relation becomes

$$
h+1 / 2 v^{2}=h_{t}
$$

and $h_{t}$ equals the total enthalpy in the freestream and is a global constant. The macroscopic chemical kinetics equation for each of the five species is

$$
\operatorname{div} \rho c_{l} v=\omega_{i}\left(\rho, T, c_{1}, \ldots, c_{5}\right)
$$

where $T$ denotes the bulk temperature of the mixture, $c_{f}$ represents the mass fraction $\rho_{k} / \rho$, and $\omega_{i}$ stands for the chemical source function of species $\ell$, i.e., the mass rate of production of species $\ell$ per unit volume by chemical reactions. Clearly, by definition, the condition

$$
\sum_{p=1}^{5} c_{l}=1
$$

must hold. Vincenti and Krueger ${ }^{2}$ develop a suitable expression for $\omega_{i}$ from principles of chemical kinetics, and the required constants and coefficients that we use are those given by Refs. 7 and 9 . The system is completed by an equation of state in two forms, one relating enthalpy $h=\left(\rho, p, c_{i}\right)$ and the other temperature $T=T\left(\rho, k, c_{i}\right)$ to density, pressure, and species concentration, the particular forms of which are considered next.

In the undisturbed flow, air is considered to be a mixture of $23.3 \%$ oxygen and $76.7 \%$ nitrogen by mass, and in the cases dealt with here its temperature behind the bow shock varies typically between 2000 and $5000 \mathrm{~K}$, seldom exceeding $6000 \mathrm{~K}$. The mixture of pure air is assumed to remain translationally and rotationally fully equilibrated while the vibrational excitation of nitric oxide and molecular oxygen

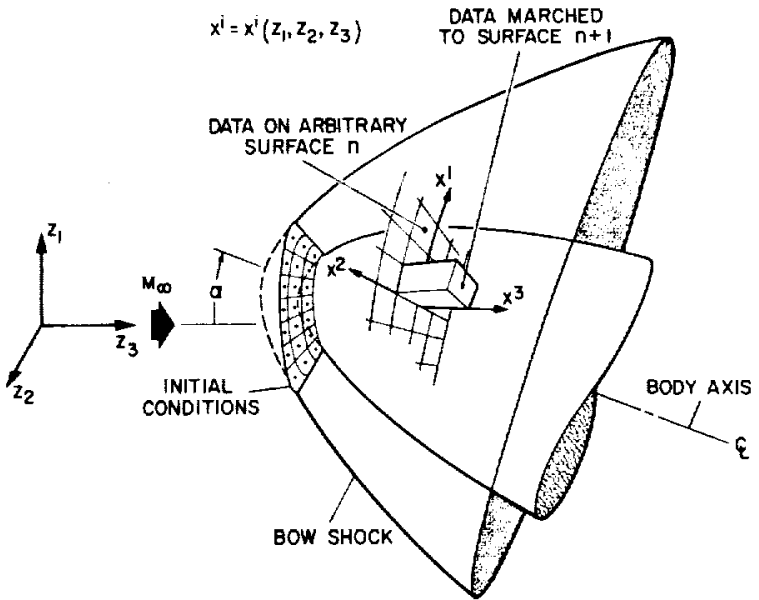

Fig. 1 Generalized coordinates and flowfield discretization for space-marching finite-volume method.

and nitrogen is approximated by the "half-excited" model of Lighthill. ${ }^{10}$ Rather than introducing nonequilibrium vibrational excitation, the vibrational mode is assumed to be excited to the extent that its energy is always half of the fully excited equilibrium value attained at high temperatures. The validity of the Lighthill model for the present application is given in Ref. 6.

The five component species are thermally perfect gases in thermal equilibrium. With the above assumptions the state equation may be written explicitly as

$$
h=\frac{\gamma}{\gamma-1} \frac{p}{\rho}+\sum_{p=l}^{5} c_{p} h_{l}^{o}
$$

where $h_{1}^{o}$ represents the net heat per unit mass evolved during the formation of species $P$ and

$$
T=p / \rho \mathbb{R} \sum_{i=1}^{s}\left(c_{i} / \mathfrak{M}_{i}\right)
$$

where the ratio of specific heats $\gamma$ is

$$
\gamma=\frac{4\left(c_{O_{2}}+c_{N_{2}}+c_{N O}\right)+5 / 2\left(c_{O}+c_{N}\right)}{2\left(c_{O_{2}}+c_{N_{2}}+c_{N O}\right)+3 / 2\left(c_{O}+c_{N}\right)}
$$

and $\mathfrak{M}_{t}$ is the molecular weight of species $\ell$ and $\mathscr{R}$ is the universal gas constant. At this point note that the fluid and thermodynamic state of the gaseous system is determined completely from the calculation of $\rho, c_{v}, v$, and $p$ satisfying the system of Eqs. (1) and (2) under specific boundary and initial conditions for a given problem.

\section{Differential and Integral Formulation}

For the method to admit initial Cauchy data situated on an arbitrary surface and march them in roughly the streamline direction, generalized coordinates must be used. The nonorthogonal curvilinear system with contravariant coordinates $x^{i}$ and covariant base vectors $g_{i}(i=1,2,3)$ is introduced where $x^{1}$ and $x^{2}$ lie in the initial data surface and $x^{3}$ is in the approximate direction of the streamlinest (Fig. 1). A reciprocal set of directions is associated with this system and is called contravariant field vectors $g^{m} \equiv \operatorname{grad} x^{m}(m=1,2,3)$ defined as the gradients of the curvilinear coordinates and related to the base vectors by $\boldsymbol{g}^{m} \cdot \boldsymbol{g}_{i}=\delta_{i}^{m}$ (Kronecker delta). Between these coordinates and a rectangular Cartesian system $z_{m}$ with unit base vectors $a_{m}$ exists the functional relationship

$$
x^{i}=x^{i}\left(z_{1}, z_{2}, z_{3}\right) i=1,2,3
$$

$\ddagger$ To minimize confusion, cxponents are not used on vectors, coordinates, or vector components; superscripts denote only the contravarient direction of the vectors and their components. 
The governing Eqs. (1) $\operatorname{div} \mathbf{J}=\operatorname{div}\left(\mathrm{Fg}_{1}+\mathbf{H g}_{2}+U \mathbf{G}_{3}\right)=\mathbf{\Omega}$ written in divergence form with respect to this curvilinear system is

$$
\frac{\partial\left(g^{1 / 2} U\right)}{\partial x^{3}}+\frac{\partial\left(g^{1 / 2} H\right)}{\partial x^{2}}+\frac{\partial\left(g^{1 / 2} F\right)}{\partial x^{I}}=\sqrt{g} \Omega
$$

where

$$
\begin{aligned}
& U= {\left[\begin{array}{c}
\rho c_{p} u^{3} \\
\rho u^{3} \\
\rho u^{3} w_{1}+p\left(\partial x^{3} / \partial z_{1}\right) \\
\rho u^{3} w_{2}+p\left(\partial x^{3} / \partial z_{2}\right) \\
\rho u^{3} w_{3}+p\left(\partial x^{3} / \partial z_{3}\right)
\end{array}\right] } \\
& \rho c_{l} u^{2} \\
& \rho u^{2} \\
& H(U)=\left[\begin{array}{c}
\rho u^{2} w_{l}+p\left(\partial x^{2} / \partial z_{l}\right) \\
\rho u^{2} w_{2}+p\left(\partial x^{2} / \partial z_{2}\right) \\
\rho u^{2} w_{3}+p\left(\partial x^{2} / \partial z_{3}\right)
\end{array}\right]
\end{aligned}
$$$$
\Omega(U)=\left[\begin{array}{c}
\omega_{i} \\
0 \\
0 \\
0 \\
0
\end{array}\right]
$$

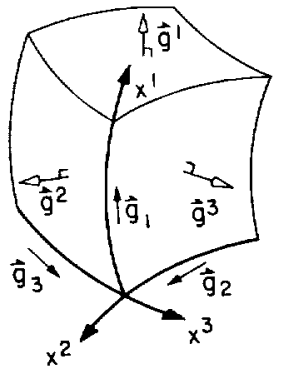

$$
F(U)=\left[\begin{array}{c}
\rho c_{i} u^{\prime} \\
\rho u^{\prime} \\
\rho u^{\prime} w_{1}+p\left(\partial x^{\prime} / \partial z_{2}\right) \\
\rho u^{\prime} w_{2}+p\left(\partial x^{\prime} / \partial z_{2}\right) \\
\rho u^{\prime} w_{3}+p\left(\partial x^{\prime} / \partial z_{3}\right)
\end{array}\right]
$$

and $g^{1 / 2}$ is the Jacobian $\partial\left(z_{1}, z_{2}, z_{3}\right) / \partial\left(x^{i}, x^{2}, x_{3}\right)$ and the flow velocity is

$$
v=\sum_{i=I}^{3} u^{i} g_{i}=\sum_{m=I}^{3} w_{m} a_{m}^{\S}
$$

A more physical interpretation of the contravarient coordinates and covariant directions can be achieved by using Gauss' theorem to cast Eq. (4) into integral or, as we call it, finite-volume form

$$
\begin{aligned}
\int_{s_{m}} J \cdot \hat{\eta} \mathrm{d} s & =\int_{s_{3}} \int U \mathrm{~d} s+\int_{s_{2}} \int H \mathrm{~d} s \\
& +\int_{s_{l}} \int F \mathrm{~d} s=\iint_{V} \int \Omega \mathrm{d} \text { vol }
\end{aligned}
$$

where $S_{m}=S g^{m}$ is one of the six surfaces (with normal $g^{\prime \prime}$ ) of the hexahedron formed by the coordinate surfaces $x^{m}=$ constant as illustrated in Fig. 2. Equations (4) and (5) are mathematically equivalent, but each has its particular advantage. The numerical analysis of a method's accuracy and stability is easier to execute with the partial differential form (4), whereas the actual computations are more conveniently carried out in the form (5); the latter is true because use of Eqs. (4) requires specifying the coordinate transformation (3) and calculating either analytically or numerically the Jacobian $g^{1 / 2}$ and the term $\partial x^{i} / \partial z_{m}$ at each cell, which for practical body shapes may be time consuming and awkward. These two quantities, however, have a geometrical interpretation that becomes obvious in the finite-volume form. For example, $\partial x^{i} / \partial z_{m}=g^{i} \cdot a_{m}$ is simply the direction cosine between contravarient direction $i$ and Cartesian direction $m$ and may be calculated from simple vector relations without having to perform any partial differentiation. Similarly, the areas and volume which appear in Eq. (5), and are evaluated by differential expressions such as $\mathrm{d} s^{i}=g^{1 / 2} \mathrm{~d} x^{2} \mathrm{~d} x^{3}$ and $\mathrm{d}$ vol $=$ $g^{1 / 2} \mathrm{~d} x^{\prime} \mathrm{d} x^{2} \mathrm{~d} x^{3}$, may also be calculated from geometrical principles. 9 The integral or finite-volume form in effect replaces explicit use of these functions with the products of vector quantities that have a definite physical significance and

\$Note that expressing the vector $\mathrm{J}=\mathrm{Fg}_{1}+\mathrm{Hg}_{2}+\mathrm{Ug}_{3}$ involving products of mixed velocity components $u^{i}$ and $w_{m}$ of both bases $g_{i}$ and $a_{m}$ leaves Eq. (4) in strong conservation form. See 8 and 11 for further discussion of this point.

The area of each quadrilateral face of the hexahedron is one half of the vector cross product of the two diagonals on that face. The hexahedron itself is composed of five tetrahedra whose volumes are computed by a simple algebraic formula. one else who has shown the equivalence of the integral and curvilinear tensor differential forms, probably because of the classical bias for orthogonal coordinates. However, we emphasize that these two formulations are identical, as of course they must be since they have the same governing equations; consequently, the accuracy and stability criteria of a chosen difference scheme when applied to one are exactly the same criteria when applied to the other. The only difference between the two forms is the way in which the coordinate transformation terms are evaluated. The differential form is very useful for establishing the physical and numerical domains of dependence which determine the condition for numerical stability. It is also vital to the analysis of the accuracy of a given numerical procedure, but it is more convenjent to carry out the actual computations using the geometrical interpretation of the finite-volume form.

\section{Numerical Procedure}

Finite-difference approximations to the governing equations are used to advance the solution in the $x^{3}$ direction from specified initial data. Split difference operators are constructed in this section to solve the finite-yolume formulation in Eq. (5) for computational cells like the one depicted in Fig. 2

\section{Difference Operators}

The essential concept of the splitting technique is to "split" the governing equation into a series of simpler component equations, so that when solved sequentially in intermediate fractional steps they approximate the original equation to some order of accuracy. For nonreacting flow, MacCormack and Paullay, ${ }^{12}$ MacCormack and Warming, ${ }^{13}$ and Rizzi, et al., ${ }^{8}$ among many others, have shown how this concept leads to splitting one spatial dimension from another; Rizzi and Bailey $^{14}$ have extended the concept to the time-dependent equations of chemically reacting flow, and, in addition, have split the chemical production term from the spatial ones which are themselves dimensionally split. We apply this idea to the steady governing Eq. (5) and break them into three difference operators

$$
\begin{aligned}
\bar{U}_{j, k}^{n+\kappa}= & \frac{1}{S^{n+1}}\left(-U_{j, k}^{n} S^{n}-F_{j, k}^{n} S_{k+1}-F_{j, k-1}^{n} S_{k}\right) \\
U_{j, k}^{n+\kappa}= & \frac{I}{2 S^{n+1}}\left(-U_{j, k}^{n} S^{n}+\bar{U}_{j, k}^{n+\kappa} S^{n+1}\right. \\
& \left.\quad-F_{j, k+1}^{n+k} S_{k+1}-F_{j, k}^{n+\wedge} S_{k}\right)
\end{aligned}
$$


$\tilde{U}_{j+k}^{n+\lambda}=\frac{I}{S^{n+l}}\left(-U_{j, k}^{n+\kappa} S^{n}-H_{j, k}^{n+k} S_{j+1}-H_{j-i, k}^{n+\kappa} S_{j}\right)$

$U_{j, k}^{n+\lambda}=\frac{I}{2 S^{n+1}}\left(-U_{j, k}^{n+\star} S^{n}+\bar{U}_{j, k}^{n+\lambda} S^{n+l}-H_{j+l, k}^{n+\lambda} S_{j+1}-H_{j, k}^{n+\lambda} S_{j}\right)$

$U_{j, k}^{n+l}=\frac{I}{S^{n+1}}\left(U_{j, k}^{n+l} S^{n}+\operatorname{vol}_{j, k}\left[I-1 / 2 \operatorname{vol}_{j, k} Q_{\pi, \rho}^{n+\lambda}\right]^{-I} \Omega_{j, k}^{n+\lambda}\right)$

where $\lambda=2 k=2 / 3$ are the fractional step numbers, and the affixal notation now no longer refers to the contravarient and covariant directions but rather to the geometrical location in the difference mesh. The subscripts $j$ and $k$ denote the discrete location of the cell $j, k$ along the axes $x^{2}$ and $x^{l}$, while $S_{j}\left(\Delta x^{3}\right)$ and $S_{k}\left(\Delta x^{3}\right)$ are the areas of its sides facing these directions. The superscripts denote the position

$$
x^{3}=\sum_{n} \Delta x^{3}(n)
$$

to which the solution has been advanced during each cycle of the operators, and $S^{n}$ is the area facing $x^{3}$. The mean values $U_{j, k}^{n}$ of the flow variables in the cell at step $n$ are defined by

$$
U_{j, k}^{n}=\frac{I}{S^{n}} \iint_{S^{n}} U \mathrm{~d} s
$$

and $F_{j, k}^{n}=F\left(U_{j, k}^{n}\right)$ and $H_{j, k}^{n}=H\left(U_{j, k}^{n}\right)$ is the usual condensed notation. The matrix $Q_{j, k}$ of the fifth order is composed of elements $\left[\left(1 / \rho u^{3} S^{n+1}\right)\left(\partial \omega_{j} / \partial c_{n}\right)\right]$ while $I$ is the identity matrix, and lastly $\left.\operatorname{vol}_{j, k}\left(\Delta x^{s}\right)\right)$ is the volume of the hexahedron enclosed by the surfaces $S_{j}, S_{j+1}, S_{k}, S_{k+1}, S^{n}$, and $S^{n+1}$. This set of equations can be written more conveniently in operator notation. Let $L,\left(\Delta x^{3}\right)$ denote the operation performed by the set of Eqs. (6a) in advancing the solution from $U_{j, k}^{n}$ to $U_{j, k}^{n+\kappa}$ (i.e., $\left.U_{j, k}^{n+\kappa}=L_{1}\left(\Delta x^{3}\right) U_{j, k}^{n}\right)$ and let $L_{2}\left(\Delta x^{3}\right)$ and $L_{\mathrm{c}}\left(\Delta x^{3}\right)$ be similarly defined by Eqs. (6b) and (6c). Analogous to the familiar MacCormack predictor-corrector scheme, the first two operators $L_{1}$ and $L_{2}$ are the two-step explicit and dimensionally split method that MacCormack and Warming ${ }^{13}$ proposed and Rizzi, et al. ${ }^{8}$ used for nonreacting flow. The last one, $L_{c}$, is an implicit modified Euler scheme that Lomax and Bailey ${ }^{15}$ have analyzed and found to be second-order accurate. Note that this last operator accounts for chemistry only, while the first two treat only convection and no chemistry. The splitting procedure, therefore, neatly segregates these two rather different phenomena.

\section{Stability Analysis}

Without any approximation, Eqs. (1) may be expressed in nondivergence form as

$$
\frac{\partial e}{\partial x^{3}}+C_{2} \frac{\partial e}{\partial x^{2}}+C_{l} \frac{\partial_{e}}{\partial x^{l}}=\Lambda(e)
$$

where $e$ is the column matrix with elements $e=\left(c_{i}, \rho\right.$, $\left.w, w_{2}, w_{3}, p\right)$. Stability conditions can be determined in the usual manner ${ }^{16}$ from an analysis of the eigenvalues of $C_{1}$ and $C_{2}$. From $L$, the condition on the step size $\delta_{l}\left(=\Delta x^{2}\right)$ in the $x^{3}$ direction is

$$
\begin{aligned}
& \delta_{l} \leq \min \\
& {\left[\frac{j, k}{\left.\left(g^{I I} / g^{33}\right)^{1 / 2}\right)\left[\nu^{\prime} \nu^{3}-c^{2} \cos \sigma+c\left(q^{2}-c^{2} \sin ^{2} \sigma\right)^{1 / 2} /\left(\nu^{3} \nu^{3}-c^{2}\right)\right.}\right]}
\end{aligned}
$$

where

$$
c^{2}=\frac{\left(\frac{\partial h}{\partial \rho}\right)_{p, c_{\ell}}}{\frac{I}{\rho}-\left(\frac{\partial h}{\partial p}\right)_{\rho, c_{\ell}}}
$$

is the square of the local frozen speed of sound, $v^{i}=v \cdot g^{i}$ / $\left(g^{i i}\right)^{1 / 2}=u^{i}\left(g^{i i}\right)^{1 / 2}$ is the component of $v$ along the $g^{i}$ direction, and

$$
g=\left(\nu^{I} \nu^{I}+\nu^{3} \nu^{3}-2 \nu^{i} \nu^{3} \cos \sigma\right)^{1 / 2}
$$

is the component of $v$ lying in the plane defined by $g^{l}$ and $\boldsymbol{g}^{3}$ and $\cos \sigma=g^{l} \cdot g^{3} /\left(g^{11} g^{33}\right)^{1 / 2}$ and

$$
g^{i i}=\sum_{m=1}^{3}\left(\partial x^{i} / \partial z_{m}\right)\left(\partial x^{i} / \partial z_{m}\right)
$$

A similar relation determining $\delta_{2}$ also exists for the $L_{2}$ operator. The chemical rate operator $L_{c}$ is implicit and a theoretical analysis using a linearized production term for $\Lambda{ }^{15}$ assures that it is unconditionally stable and hence may be controlled by either of the two explicit step sizes. The sequence of operators

$$
U_{j, k}^{n+\prime}=L_{c}(\delta) L_{2}(\delta) L_{l}(\delta) U_{j, k}^{n}
$$

representing Eqs. (6) is stable if the necessary condition

$$
\delta \leq \min \left(\delta_{l}, \delta_{2}\right)
$$

is satisfied.

\section{Accuracy Analysis}

The sequence in Eqs. (6) is known to be accurate only to the first order of $\delta$ because of the noncommutativity of the operators. A symmetric sequence, however,

$$
U_{j, k}^{n+2}=L_{1} L_{2} L_{c} L_{c} L_{2} L_{1} U_{j, k}^{n}
$$

can be constructed by reversing the cycle of operators on each successive step, and in the next paragraph we will show that second-order accuracy is regained by so doing.

Consider Eq. (8) where $e$ now is the solution vector discretized over a computational mesh or net of individual points. The partial derivative terms $C_{2}\left(\partial / \partial x^{2}\right)$ and $C_{l}(\partial /$ $\left.\partial x^{l}\right)$ are represented by the matrices $A$ and $B$ and will later be approximated by the particular difference scheme that is chosen. So far, however, the full nonlinearity of Eq. (8) remains intact, but at this time $\Lambda$ must be locally linearized by $\Lambda=W_{e}+O\left(\delta^{2}\right)$ where $W$ is the Jacobian matrix $W=\left[\partial \Lambda / \partial_{e}\right]$. Equation (8) may therefore be written as

$$
\frac{\partial e}{\partial x^{3}}=\left[-C_{1} \frac{\partial}{\partial x^{l}}-C_{2} \frac{\partial}{\partial x^{2}}+\mathrm{W}\right]_{e}=[\mathrm{A}+\mathbf{B}+\mathrm{W}]_{e}
$$

and further, if $C_{1}, C_{2}$, and $\mathrm{W}$ are constant over $x^{3}$, the second derivative in $x^{3}$ then becomes

$$
\frac{\partial}{\partial x^{3}}\left(\frac{\partial}{\partial x^{3}} c\right)=[\mathrm{A}+\mathrm{B}+\mathrm{W}]^{2} e
$$

With the aid of this last assumption the accuracy of sequence in Eq. (12) will now be analyzed on Eq. (13), although with more labor it can also be proven that the symmetric sequence in Eq. (12) maintains the same accuracy as a second-order nonsplit method for the fully nonlinear problem as well. The explicit nonsplit MacCormack scheme when applied twice to 
Eq. (13) yields the matrix equation

$e^{n+1}=\left[I+2 \delta(\mathrm{A}+\mathrm{B}+\mathrm{W})+\frac{(2 \delta)^{2}}{2}\left(\mathrm{~A}^{2}+\mathrm{B}^{2}+\mathrm{W}^{2}+\mathrm{AB}\right.\right.$

$+\mathrm{BA}+\mathrm{AW}+\mathrm{WA}+\mathrm{BW}+\mathrm{WB})] e^{n}+O\left(\delta^{3}\right)$

and is accurate to second order because it matches the Taylor series expansion of e through order $\delta^{2}$. In a similar way the sequence (10) has the matrix representation

$\left(I-\frac{I}{2} \delta \mathrm{W}\right) e^{n+l}=\left(I+\frac{I}{2 \delta} \mathrm{W}\right)\left(I+\delta \mathrm{B}+\frac{\delta^{2}}{2} \mathrm{~B}^{2}\right)\left(I+\delta \mathrm{A}+\frac{\delta^{2}}{2} A^{2}\right) e^{n}$

or

$$
\begin{aligned}
e^{n+I}= & {\left[I+\delta\left(\mathrm{A}+\mathrm{B}+\mathrm{W}+\frac{\delta^{2}}{2}\left(\mathrm{~A}^{2}+B^{3}+\mathrm{W}^{2}+2 \mathrm{BA}+2 \mathrm{WB}\right)\right] e^{n}\right.} \\
& +O\left(\delta^{3}\right)
\end{aligned}
$$

and because of the noncommutativity of the matrices the terms multiplying $\delta^{2}$ do not equal those in Eq. (14). Hence, the sequence is accurate only to the first order. Reversing the cycle for the next step, however, yields

$$
\begin{aligned}
& e^{n+2}=\left[I+\delta(\mathrm{W}+\mathrm{B}+\mathrm{A})+\frac{\delta^{2}}{2}\left(\mathrm{~W}^{2}+\mathrm{B}^{2}+\mathrm{A}^{2}+2 \mathrm{BW}+2 \mathrm{AW}\right.\right. \\
& +2 \mathrm{AB})]\left[I+\delta(\mathrm{A}+\mathrm{B}+\mathrm{W})+\frac{\delta^{2}}{2}\left(A^{2}+B^{2}+W^{2}+2 \mathrm{BA}\right.\right. \\
& \quad+2 \mathrm{WB}] \mathrm{e}^{n}+O\left(\delta^{3}\right) \\
& =\left[I+2 \delta(\mathrm{A}+\mathrm{B}+\mathrm{W})+\frac{(2 \delta)^{2}}{2}\left(\mathrm{~A}^{2}+\mathrm{B}^{2}+\mathrm{W}^{2}+\mathrm{AB}\right.\right. \\
& +\mathrm{BA}+\mathrm{AW}+\mathrm{WA}+\mathrm{BW}+\mathrm{WB})] \mathrm{e}^{n}+O\left(\delta^{3}\right)
\end{aligned}
$$

which is identical to the unsplit expression in Eq. (14) and therefore the sequence (12) of mixed explicit and implicit operators in Eq. (6) is accurate to second order in space.

\section{Multiple Chemical Steps}

Splitting the difference scheme does not degrade its order of accuracy, instead some advantages do accrue. Not only does it simplify the programming but MacCormack and Warming ${ }^{13}$ have pointed out that splitting the spatial dimensions increases the computational efficiency by allowing larger integration steps. The greatest advantage, however, is gained from separating the fluid dynamical phenomena from those of chemical kinetics because each of these has intrinsically different rates which they proceed. The convection process, for example, is slowly varying in space so that a relatively coarse step $\delta$ can accurately resolve its variation in $x^{3}$, whereas in comparison, the chemistry is much more rapidly changing and cannot be determined accurately through use of the same coarse step $\delta$ even though the calculation is stable for any $\delta$.

To increase the resolution in this case we use a selfcontrolling increment routine that takes several fractional steps of $\delta_{c}$ for each coarse one of $\delta$ and that, following Lomax and Bailey, ${ }^{15}$ checks at each step whether the last integration has produced a change in $c_{i}$ for the next partial step, and diminishes it if it has, so that in effect it always tries to advance with a step size that yields a $10 \%$ change in the solution. From our experience in the cases presented here, the chemistry resolution typically requires five intermediate steps for each one for the transport process, thus severely increasing the computational time. Some workers ${ }^{6,7}$ have coped with this burden by solving the chemical rate equation [ first row of Eq. (4)] separately from the others. In their approach they ad-



Fig. 3 Depiction of the variable-step procedure for $L_{c}$. Several of the smaller chemical steps $\delta_{c}$, malch one single larger convective slep $\delta$.

vance the gas dynamic equations at the usual CFL condition and then, with mixed explicit and implicit differences, return to integrate the rate equation using a variable step size similar to ours.

While this intuitive procedure seems to give satisfactory results, it is difficult to determine its order of accuracy and stability criteria. Segregating the fluid and chemical phenomena by using the split operators in Eqs. (6) in the sequence

$$
U_{j, k}^{n+2}=L_{l}(\delta) L_{2}(\delta) \prod_{i=1}^{E} L_{c}\left(\delta_{c}\right) L_{2}(\delta) L_{l}(\delta) U_{j, k}^{n}
$$

employing various chemical steps $\delta_{c}$ so that

$$
\sum_{i=1}^{E} \delta_{c^{\prime}}=2 \delta
$$

is a more theoretically sound way of dealing with their different rates. First it is more efficient because it allows all of the fluid to be convected by operators in Eqs. (6a) and (6b) in one single, large increment $\delta$, and then remain idle during the time the chemical operator $L_{c}$ advances repeatedly at the smaller rate $\delta_{c}$ in order to "catch up" to the flow at $\delta$ (see Fig. 3 ). While in effect this is similar to what other methods do 6,7 it is not identical because those methods advance the chemical rate equation which contains some convection terms that are differenced explicitly. Our $L_{c}$ contains no convection terms and hence eliminates the need for computing these differences as well as applying their boundary conditions at all intermediate points. Furthermore, in contrast to the chemical rate equation, the split operator $L_{c}$ can be differenced easily with one implicit scheme that is unconditionally stable. Splitting avoids the possible danger of restricted stablity that Lomax and Bailey ${ }^{15}$ warn us can arise with use of mixed difference expressions.

\section{Computational Mesh Construction}

In order for the sequence just described to advance the solution, the method must first construct the coordinate mesh network over which to integrate the equations. The coordinate system $\left(x^{l}, x^{2}, x^{3}\right)$, which may be nonorthogonal and unequispaced, is formed by three families of intersecting surfaces (Fig. 4). The first one constructed is an arbitrarily oriented surface $x^{3}=$ constant at the $n+I$ step, constrained only by the CFL condition on $\delta$, and it intersects a second family $x^{2}=$ constant that at each extremity coincides with the shock ${ }^{* *}$ and the body surface and varies in some prescribed manner in between. The last family, $x^{\prime}=$ constant, is composed of simple planes and intersects the second at a number of given angular increments of $\phi$. This new marching surface

\footnotetext{
"How to determine the location of the shock wave at the new step is explained in the next section.
} 


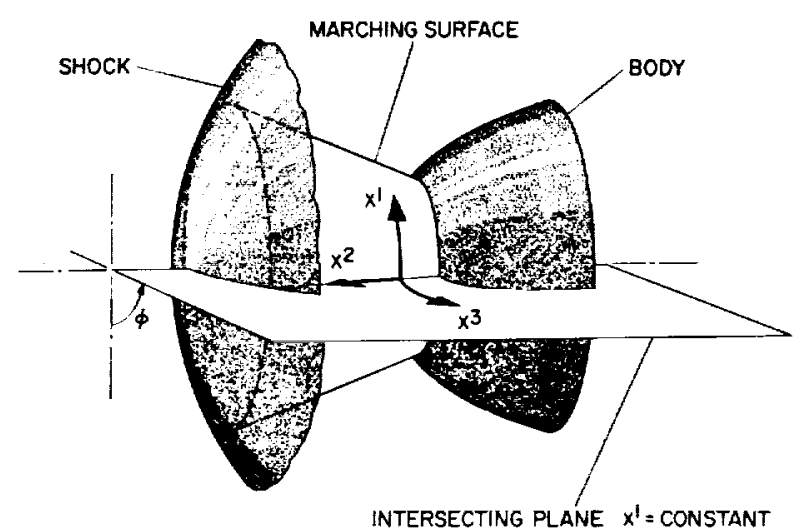

Fig. 4 Definition of general coordinates $x^{\prime}, x^{2}, x^{3}$ by the inlersection of three surfaces.

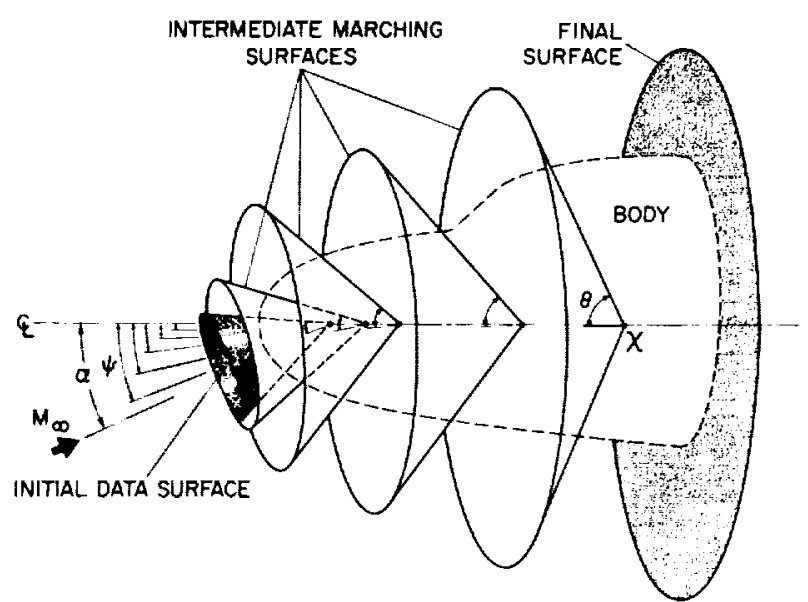

Fig. 5 Illustration of position, orientation, and vertex angle of conical data surfaces for successive steps.

at $n+I$ can be completely general, and for the cases presented here we have chosen it to be circular conical because this requires at every step only one parameter for each of the three aspects of general motion. Translation of the cone is accounted for by the location $\chi$ of its apex along the body axis, rotation by the angle $\psi$ between its axis and the body's, and, lastly, dilatation by its vertex angle $\theta$, as illustrated in Fig. 5 . The second series of surfaces, of which the first and last are the body and shock, partition each cone into a number of contiguous annular segments. Each of these is then intersected by a plane rotated about the cone axis in specified angular increments. The resulting intersections delineate the coordinate $x^{2}$ and are straight lines or rays and complete the definition of the coordinates $x^{4}, x^{2}, x^{3}$. As the solution proceeds downstream, $x$ advances while $\theta$ increases monotonically toward $90^{\circ}$ and $\psi$ approaches zero so that at the final step the surface $x^{3}=$ constant degenerates into a plane normal to the body axis.

\section{Shock Location and Boundary Conditions}

The intersections of the coordinates $x^{3}$ and $x^{2}$ lying in the marching surface $x^{3}=$ constant map out a mesh network of small quadrilateral cells in such a way that the innermost row lies on the body and the outermost row is aligned with the shock. This method, termed mesh aligning, has the following features. For each cell at the outer edge of the mesh, the pressure $p_{s}$ just downstream of the bow wave specifies the inclination $\hat{\eta}_{s}=g^{2}$ of the shock through the steady shock relations. The surface whose local slope matches this at each cell is constructed, and it becomes the outer edge of the mesh for the next step. Although the pressure $p_{s}$ at the shock is unknown it can be calculated (see Fig. 6) from the interior

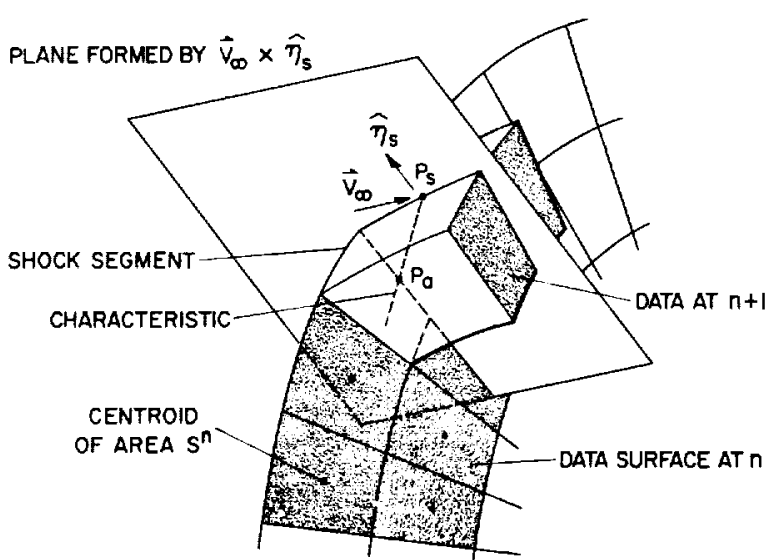

Fig. 6 Determination of the bow shock surface using a characteristic relation.

values $p_{a}$ by solving for the two unknowns, $p_{s}$ and $\tilde{\psi}_{s}$, simultaneously and one local characteristic equation valid in the plane defined by the freestream $v_{\infty}$ and $\hat{\eta}_{s}$. The solution at each cell is carried out rapidly by an iterative procedure.

Thus at the outermost row of cells $\boldsymbol{g}^{2}$ becomes identical to the outward normal $\hat{\eta}_{s}$ and to $\hat{\eta}_{b}$ at the body surface. This situation simplifies imposition of the conditions at the outer and inner edges of the overall mesh, referred to as entrance (shock wave) and streamline (body) boundaries. Along the entrance boundary the flow variables are held fixed at their supersonic freestream values. Without modification, the difference operators (6) then conserve mass and momentum across the discontinuity. This is precisely the condition used to derive the Rankine-Hugoniot relations, however, so that in effect, application of the difference operators across the shock reduces numerically to the analytic jump conditions. Across cell faces that lie on the body, no transport is allowed. The only nonzero variable actually needed at that point in the calculations is the pressure $p_{b}$, which is related to the flow properties in adjacent interior cells by a characteristic relation in a manner similar to that at the shock wave.

\section{Results}

Computed results for reacting airflow about a general body traveling at $6.54 \mathrm{~km} / \mathrm{sec}$, Mach number $M_{\infty}=21.7$, and $41^{\circ}$ angle of attack are presented. The ambient freestream pressure is $p_{\infty}=106 \mathrm{dynes} / \mathrm{cm}$, the temperature is $T_{\infty}=236 \mathrm{~K}$, and the body is a smooth three-dimensional configuration described by a series of third-degree polynomials in plan and profile views and ellipses in cross section. Between the body and shock the mesh holds nine cells and around the body in the meridional direction there are seventeen.

The trace of the bow shock in the plane of symmetry, along with cross-sectional views at three axial locations, are displayed in Fig. 7, and clearly illustrate the large asymmetry of the flow at this high angle of attack. At cross section B-B the wing of the spacecraft is beginning to protrude out from the fuselage and grows steadily larger downstream. Figure 8 presents the radial distribution of atomic oxygen $c_{O}$ and nitric oxide $\mathrm{c}_{\mathrm{NO}}$ in both the windward and leeward parts of the symmetry plane at the three designated axial stations. The high temperature near the windward portion of the shock wave sharply enhances the concentration $c_{O}$ of atomic oxygen which then quickly levels off nearer to the body. On the lee side, however, the shock temperature is much lower and the main rise in $c_{0}$ occurs halfway between the shock and body, presumably due to convective transport from the windward region. For all axial positions, though, the same maximum value is reached at the vehicle surface suggesting that the flow is probably frozen there. The concentration $\mathrm{c}_{\mathrm{NO}}$ of nitric oxide also increases dramatically to a maximum directly behind the windward portion of the bow wave and then falls 


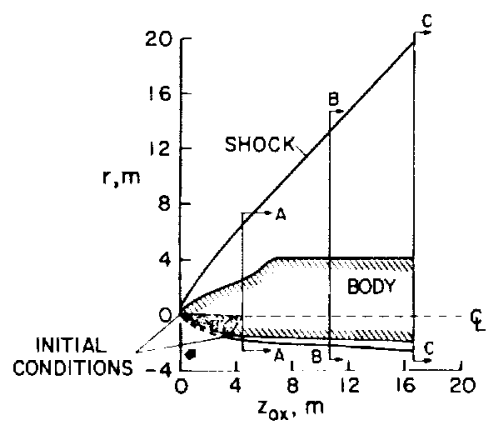

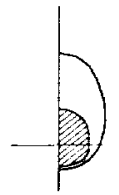

SECTION A-A $z_{a x}=4.4 m$

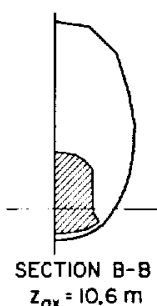

$z_{a x}=10.6 \mathrm{~m}$



Fig. 7 Bow shock shape for reacting flow past a three-dimensional body traveling at velocity $\nu_{\infty}=6.54 \mathrm{~km} / \mathrm{sec}$, Mach number $M_{\infty}$ $=21.7$, temperature $T_{\infty}=236 \mathrm{~K}$, pressure $p_{\infty}=106 \mathrm{dynes} / \mathrm{cm}^{2}$, and angle of attack $=41^{\circ}$.

rapidly to a minimum at the spacecraft. On the lee side $\mathrm{c}_{\mathrm{NO}}$ is small at the shock, and the maximum is achieved roughly midway to the vehicle. The overall nature of the species distribution is typified in Fig. 9 by the circumferential variation of $\mathrm{c}_{\mathrm{O}}$ and $\mathrm{c}_{\mathrm{NO}}$ along the shock and body in the plane $Z_{a r}=16.6 \mathrm{~m}$. Since the bow shock is hottest on the windward side, the production of both $\mathrm{O}$ and $\mathrm{NO}$ is also largest there. Approaching leeward the shock becomes progressively weaker, and the concentrations of both $O$ and NO fall precipitously. The same trend, however, does not occur at the body. Although $\mathrm{c}_{\mathrm{NO}}$ attains a maximum at about $70^{\circ}$ where the wing protrudes, $c_{O}$ remains curiously constant at a maximum value around the entire vehicle even though its production at the lee region of the shock is very low. This indicates that local chemical production plays no role here. Rather, the convection process predominates; that is, atomic oxygen is produced at the windward part of the bow shock and is swept around to the lee side of the body. The vehicle is in an environment of greatly dissociated oxygen.

For exactly the same flow as in Fig. 7, except that now $\alpha=$ $30^{\circ}$, two temperature plots in the windward symmetry plane are presented in Fig. 10 and compared with the results from a method of characteristics computation by Rakich et al. ${ }^{9}$ that are denoted by solid dots. The first part of Fig. 10 presents the axial variation along the body surface and the second at the radial distribution between body and shock at $z_{a x}=15.7 \mathrm{~m}$. Both indicate that temperature is a rather slowly changing property, usually in the range between $4000 \mathrm{~K}$ and $6000 \mathrm{~K}$, although interestingly there is a sharp rise of about $1000 \mathrm{~K}$ near the body. Rakich et al. found a similar effect and attributed it to an "entropy layer" around the body.

\section{Concluding Remarks}

The method presented demonstrates that nonorthogonal curvilinear coordinates can be useful for computing practical fluid problems and in fact are the underlying basis of the integral or finite-volume form of the governing equations. Unlike other marching methods, the finite-volume approach offers a wider choice of surfaces upon which the initial conditions may lie, and it allows the solution to advance in an ar-
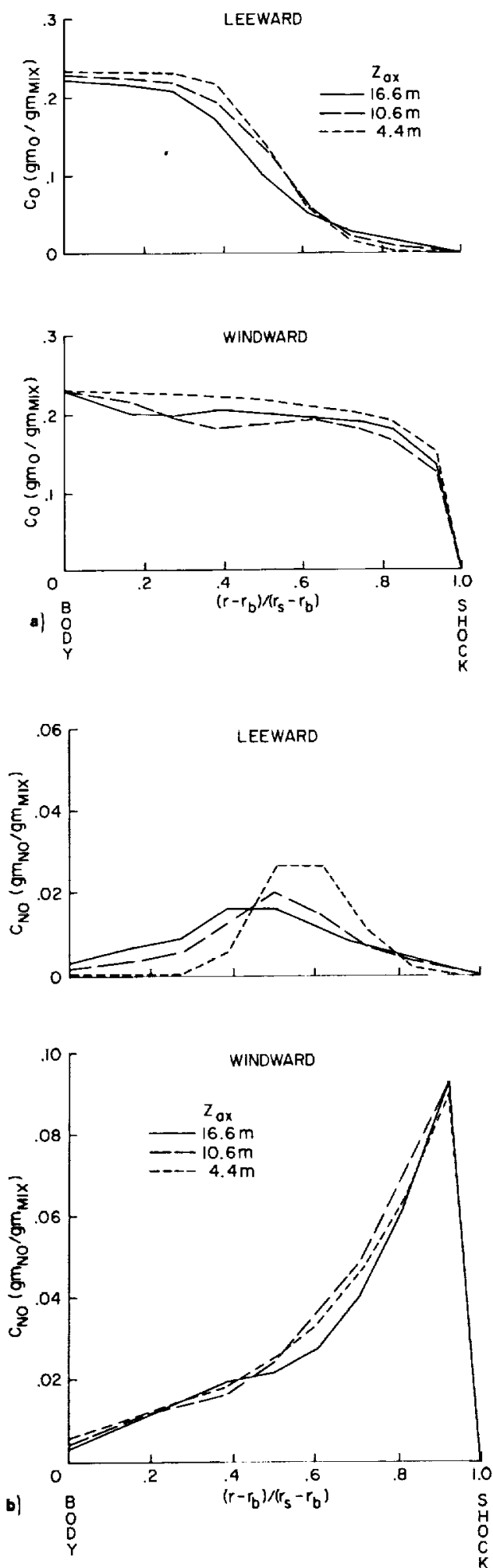

Fig. 8 Radial distribution of the chemical species $O$ and NO in the windward and leeward sides of the symmetry plane for the flow in Fig. 7. a) Radial distribution of $\mathrm{O}$; b) Radial distribution of NO

bitrary direction while at the same time it simplifies application of the body boundary conditions. Furthermore, this procedure lends itself to a new scheme of aligning the mesh with the bow shock, which is simple to implement, accurate, and consistent with the interior flow. Using the fractional step routine does not lower a difference scheme's order of accuracy, but rather several benefits are gained. Split difference operators prove to be very appropriate for handling the di- 

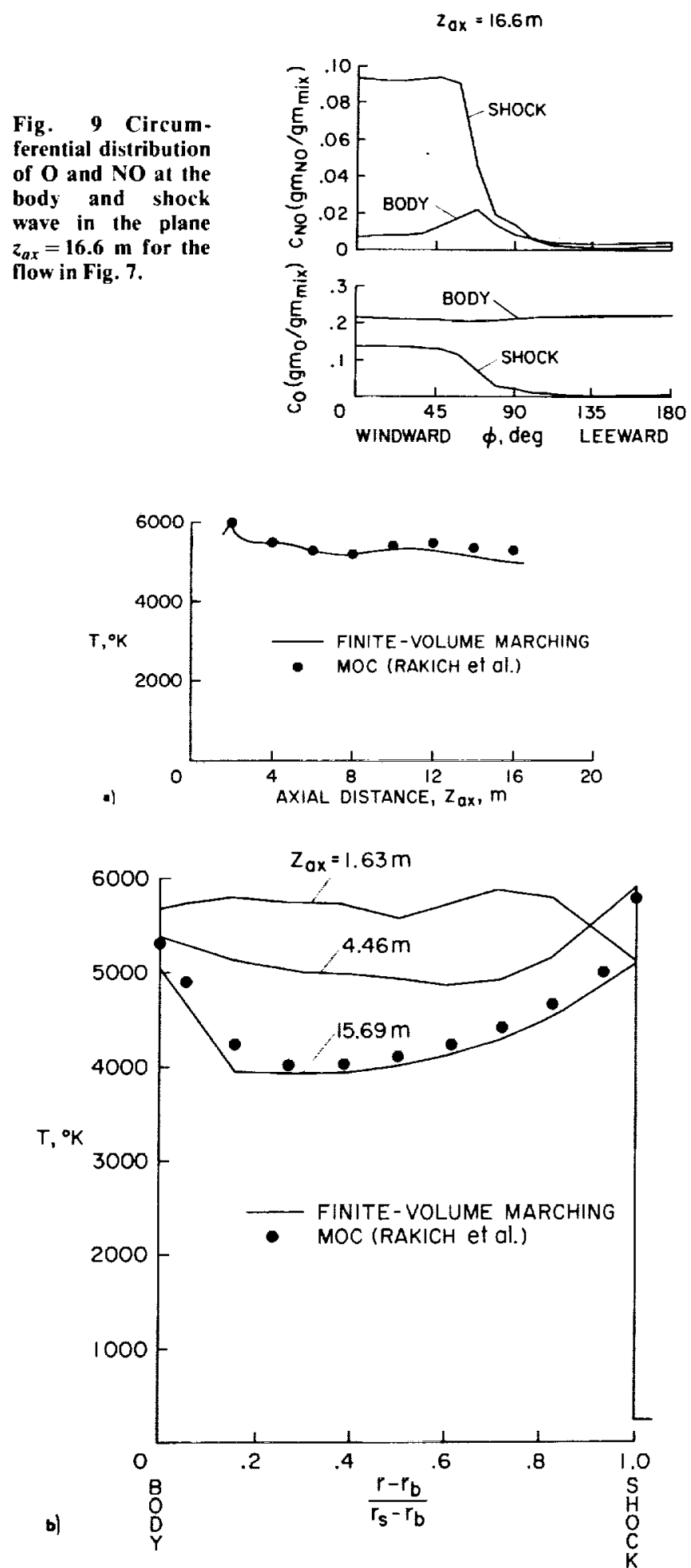

Fig. 10 Temperature plots in the wind ward symmetry plane for flow identical to that in Fig. 7 except that now $\alpha=30^{\circ}$; a) Axial variation along the body surface; b) Radial variation between body and shock.

verse nature of the chemical kinetics and gas-dynamics phenomena. In particular, they provide a natural way of mixing explicit and implicit difference schemes that may use differing step sizes, and at the same time ease the computational burden. Results from this method are in accord with those from a characteristics procedure. Furthermore, a check on the conservation of total oxygen and nitrogen atoms, expressions independent of the calculations, reveals an overall error of only about $1 \%$. The computation for the case presented terminates at about $20 \mathrm{~m}$ because the shock wave generated by the leading edge of the wing collides with the bow wave and appears to produce a small pocket of subsonic flow at that point. The space-marching finite-volume method, of course, is restricted to supersonic flow and another procedure would be required to compute this phenomenon.

\section{References}

'Spurk, J.H., Gerber, N., and Sedney, R., "Characteristics Calculation of Flowfields with Chemical Reactions," AIAA Journal, Vol. 4, Jan. 1966, pp. 30-37.

${ }^{2}$ Vincenti, W.G. and Kruger, C.H., Introduction to Physical Gas Dynamics, Wiley, New York, 1965.

${ }^{3}$ Der, J.J., "Theoretocai Studies of Supersonic Two-Dimensional Axisymmetric Nonequilibrium Flow, Including Calculations of Flow through a Nozzle," TR R-164, 1963, NASA

${ }^{4}$ Kyriss, C.L." "A Time Dependent Solution for the Blunt Body Flow of a Chemically Reacting Gas Mixture," AIAA Paper 70-771, Los Angeles, Calif., 1970.

${ }^{5}$ Taylor, A.A. and Hoffman, J.D., "Design of Maximum Thrust Nozzles with Nonequilibrium, Chemically Reacting Flow," AFAPLTR-71-92, Air Force Aero Propulsion Lab., Wright Patterson Air Force Base, Ohio.

${ }^{6}$ Rakich, J.W. and Park, C., "Nonequilibrium Three-Dimensional Supersonic Flow Computations with Application to the Space Shuttle Orbiter Design," Symposium on Application of Computers to Fluid Dynamic Analysis and Design, Polytechnic Institute of Brooklyn Grad. Center, Farmingdale, N.Y., Jan. 3-4, 1973.

'Davy, W.C. and Reinhardt, W.A." "Computation of Shuttle Nonequilibrium Flow Fields on a Parallel Processor,"' Proceedings of the NASA Conference on Aerodynamic Analysis Requiring Advanced Computers, NASA SP347, 1975.

${ }^{8}$ Rizzi, A.W., Klavins, A., and MacCormack, R.W., "A Generalized Hyperbolic Marching Technique for Three-Dimensional Supersonic Flow with Shocks," Proceddings of the Fourth International Conference on Numerical Methods in Fluid Dynamics, ed., Robert D. Richtmyer, Lecture Notes in Physics, 35, Berlin, Springer-Verlag, 1976.

${ }^{9}$ Rakich, J.V., Bailey, H.E., and Park, C., "Computation of Nonequilibrium Three-Dimensional Inviscid Flow Over Blunt Nosed Bodies Flying at Supersonic Speeds," AIAA Paper 75-835, Hartford, Conn., 1975.

${ }^{10}$ Lighthill, M.L., "Dynamics of a Dissociating Gas, Journal of Fluid Mechanics, Vol. 2, Jan. 1957, pp. 1-32.

${ }^{11}$ Vinokur, M., "Conservation Equations of Gas-dynamics in Curvilinear Coordinate Systems," Journal of Computational Physics, Vol. 14, Feb. 1974, pp. 105-125.

${ }^{12}$ MacCormack, R.W. and Paullay, A.J., "Computational Efficiency Achieved by Time Splitting of Finite-Difference Operators," AIAA Paper 72-154, San Diego, Calif., 1972.

${ }^{13}$ MacCormack, R.W. and Warming, R.F., "Survey of Computational Methods for Three-Dimensional Supersonic Inviscid Flows with Shocks," Advances in Numerical Fluid Dynamics, AGARDograph, 64, 1973.

${ }^{14}$ Rizzi, A.W. and Bailey, H.E., "Reacting Nonequilibrium Flow around the Space Shuttle Using a Time Split Method," Proceedings of the NASA Conference on Aerodynamic Analysis Requiring Advanced Computers, NASA SP-347, 1875 .

${ }^{15}$ Lomax, H. and Bailey, H.E., "A Critical Analysis of Various Numerical Integration Methods for Computing the Flow of a Gas in Chemical Nonequilibrium." TN D-4109, 1967, NASA.

${ }^{16}$ Richtmyer, R.D. and Morton, K.W., 'Difference Methods for Initial-Value Problems, 2nd ed., Interscience, New York, 1967. 